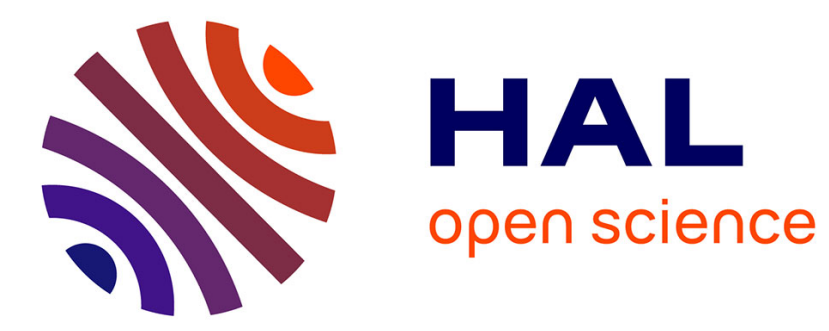

\title{
Bright, low debris, ultrashort hard x-ray table top source using carbon nanotubes
}

S. Bagchi, P.P. Kiran, K. Yang, A.M. Rao, M.K. Bhuyan, M. Krishnamurthy, G.R. Kumar

\section{- To cite this version:}

S. Bagchi, P.P. Kiran, K. Yang, A.M. Rao, M.K. Bhuyan, et al.. Bright, low debris, ultrashort hard x-ray table top source using carbon nanotubes. Physics of Plasmas, 2011, 18 (1), pp.014502. 10.1063/1.3531685. hal-00611037

\section{HAL Id: hal-00611037 \\ https://hal.science/hal-00611037}

Submitted on 13 May 2021

HAL is a multi-disciplinary open access archive for the deposit and dissemination of scientific research documents, whether they are published or not. The documents may come from teaching and research institutions in France or abroad, or from public or private research centers.
L'archive ouverte pluridisciplinaire $\mathbf{H A L}$, est destinée au dépôt et à la diffusion de documents scientifiques de niveau recherche, publiés ou non, émanant des établissements d'enseignement et de recherche français ou étrangers, des laboratoires publics ou privés. 


\title{
Bright, low debris, ultrashort hard x-ray table top source using carbon nanotubes
}

\author{
Suman Bagchi, ${ }^{1,2}$ P. Prem Kiran, ${ }^{1,2}$ K. Yang, ${ }^{3}$ A. M. Rao, ${ }^{3}$ M. K. Bhuyan, ${ }^{4}$ \\ M. Krishnamurthy, ${ }^{1}$ and G. Ravindra Kumar ${ }^{\top}$ \\ ${ }^{1}$ Tata Institute of Fundamental Research, 1 Homi Bhabha Road, Mumbai 400005, India \\ ${ }^{2}$ Advanced Centre of Research in High Energy Materials (ACRHEM), University of Hyderabad, \\ Hyderabad 500046, India \\ ${ }^{3}$ Department of Physics and Astronomy, Clemson University, Clemson, South Carolina 29634, USA \\ ${ }^{4}$ Department d'Optique P. M. Duffieux, Institut FEMTO-ST, CNRS UMR 6174, \\ Universite de Franche-Comte, 25030 Besancon, France
}

(Received 6 July 2010; accepted 2 December 2010; published online 11 January 2011)

\begin{abstract}
We demonstrate that carbon nanotube coated surfaces produce two orders of magnitude brighter hard x-ray emission, in laser produced plasmas, than planar surfaces. It is accompanied by three orders of magnitude reduction in ion debris which is also low $Z$ and nontoxic. The increased emission is a direct consequence of the enhancement in local fields and is via the simple and well known "lightning rod" effect. We propose that this carbon nanotube hard x-ray source is a simple, inexpensive, and high repetition rate hard x-ray point source for a variety of applications in imaging, lithography, microscopy, and material processing. (c) 2011 American Institute of Physics.

[doi:10.1063/1.3531685]
\end{abstract}

Currently major efforts are being expended on tailoring plasma X-ray sources produced by ultrashort laser pulses. Typical targets are planar solids, ${ }^{1-3}$ though microsphere, ${ }^{4}$ nanoparticle ${ }^{5}$ coated surfaces, as well as microdroplets ${ }^{6}$ and gaseous clusters ${ }^{7-9}$ are beginning to be explored. While such sources offer great promise-particularly in the hard $\mathrm{x}$-ray region-their efficiencies are low (typically ${ }^{10} 10^{-7}$ at $10^{17} \mathrm{~W} \mathrm{~cm}^{-2}$ ) and the laser intensity required is still quite high $\left(>10^{16} \mathrm{~W} \mathrm{~cm}^{-2}\right)$; the latter factor limits the repetition rates of these sources. There is a great and urgent need to find compact, high repetition rate, low laser intensity $\left(10^{14}-10^{15} \mathrm{~W} \mathrm{~cm}^{-2}\right)$ excited plasma sources that can be readily used for various scientific and technological applications. Carbon nanotubes ${ }^{11}$ have been convincingly shown to be efficient emitters of electrons ${ }^{12}$ at low applied voltages. The behavior is well explained by simple electrostatic theory ${ }^{13}$ and obeys Fowler-Nordheim behavior ${ }^{14}$ under dc and pulsed excitations. ${ }^{15}$ Earlier work ${ }^{16}$ has shown increased soft $\mathrm{x}$-ray emission from laser plasmas created on carbon nanotube coated targets. In addition, they are good for generating ultrashort electron bunches with proven ability to generate x-rays. ${ }^{17}$ These observations spark the idea of exploiting the propensity for electron emission of carbon nanotubes to design more efficient laser produced plasma $\mathrm{x}$-ray sources.

We used multiwalled carbon nanotubes (MWNTs) that were synthesized on copper substrates through the catalytic pyrolysis of a ferrocene-xylene mixture at $675{ }^{\circ} \mathrm{C}$ fed into a tubular two-stage quartz reactor. ${ }^{18}$ Scanning electron microscope (SEM) images reveal that the MWNTs have a length of $1.1 \mu \mathrm{m}$ and diameter of $0.1 \mu \mathrm{m}$ with a typical areal density of $3 \times 10^{9} \mathrm{~cm}^{-2}$. Experimental studies ${ }^{19}$ (Fig. 1) were performed with $p$-polarized, $50 \mathrm{fs}, 800 \mathrm{~nm}, 10 \mathrm{~Hz}$ Ti: sapphire laser (THALES, ALPHA 10) pulses. To avoid any detrimental preionization effects because of the nanosecond prepulse, at an intensity of about $10^{16} \mathrm{~W} \mathrm{~cm}^{-2}$, contrast ratio was carefully kept at a level better than $10^{-6}$. The typical amplified spontaneous duration was 5-8 ns. The laser pulses were focused on to the target at an incident angle of $23^{\circ}$ with respect to target normal direction by an off axis parabolic mirror to focal spot size of $10 \mu \mathrm{m}\left(1 / e^{2}\right)$, as determined by an equivalent imaging technique. Both the targets were mounted adjacently in a vacuum chamber with a base pressure of $10^{-6}$ Torr. A motorized stage assembly was used to ensure those fresh target portions were exposed to each laser irradiation. Measurements of hard x-ray emission in the 50$500 \mathrm{keV}$ range were performed with a calibrated $\mathrm{NaI}(\mathrm{Tl})$ detector. ${ }^{20}$ The NaI (Tl) detector was (a) gated in time with respect to incident laser pulse to ensure background free data acquisition and (b) covered by thick lead walls and lead apertures to reduce the probability of recording "piled up" events. The signals from the detector were collected by a multichannel analyzer attached to a computer. The x-ray yield was collected over 128 laser shots at each laser intensity. The energy of the ions emitted along the target normal direction from the plasma was measured by a channel electron multiplier (CEM) device, used in proportional mode, exploiting conventional ion arrival time measurement technique. Four large annular Faraday cups ${ }^{19}$ (AnFCs) were placed at angles of $5^{\circ}$ (FC 1), $8^{\circ}$ (FC 2), $12^{\circ}$ (FC 3), and $17^{\circ}$ (FC 4) with respect to target normal direction to measure the angular distribution of the emitted ion flux.

SEM images of optically polished copper targets $(\mathrm{Cu})$ and optically polished copper targets coated with MWNT are shown in Figs. 1(b) and 1(c). Figure 1(d) shows a high resolution SEM image of an individual MWNT. It is clear from Figs. 1(b) and 1(d) that the nanotubes are aligned vertical to the surface. As previously established, ${ }^{16}$ such alignment facilitates $\mathrm{x}$-ray emission from the laser produced plasma. Femtosecond laser excited $\mathrm{x}$-ray and ion emissions from 


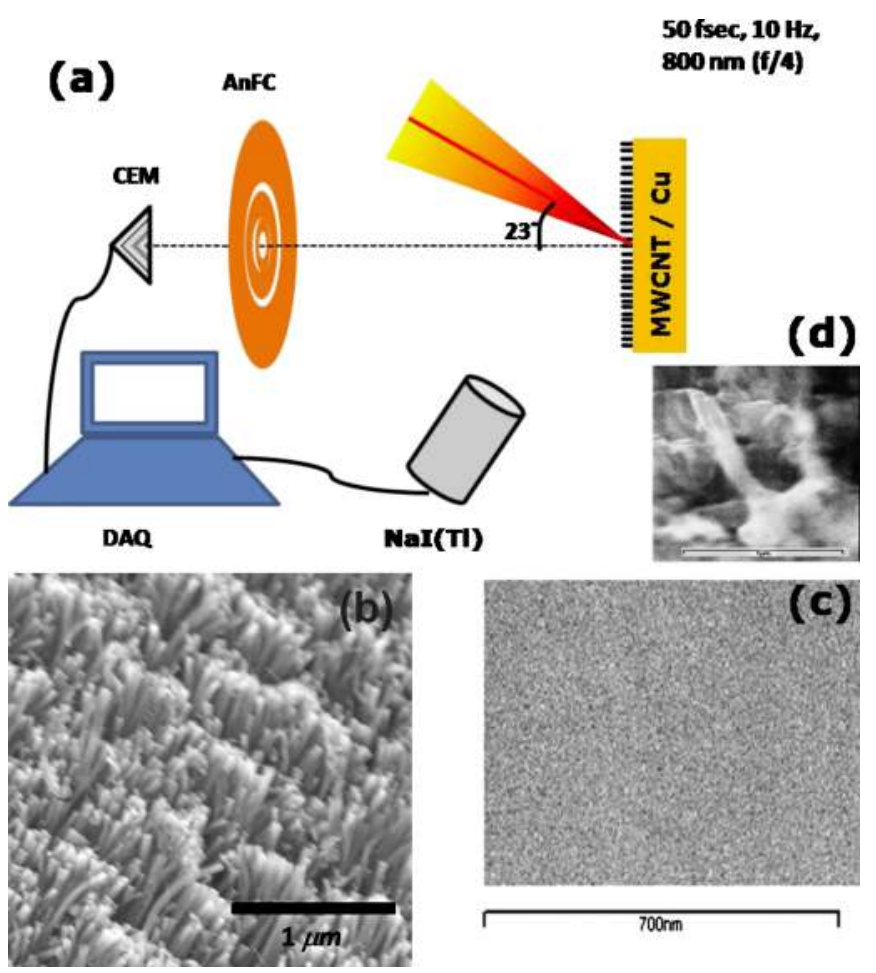

FIG. 1. (Color online) Schematic experimental arrangement is shown in (a). The ion energies along the target normal direction are measured using CEM, and AnFCs are employed to estimate the angular divergence of the ion emission. SEM image (top view) of the MWNT and polished copper $(\mathrm{Cu})$ are shown in (b) and (c), respectively. A SEM image of an individual MWNT is shown in (d).

MWNT coated targets are compared with polished targets to establish the enhancement/suppression factors.

Comparison of bremsstrahlung emission yields clearly reveals that MWNT (black open square) samples produce two orders of magnitude more $\mathrm{x}$-ray flux than $\mathrm{Cu}$ (red open circles) over most of the laser intensity range considered in the experiment (see Fig. 2). A typical bremsstrahlung spectrum is shown in Fig. 2(c). A nonlinear increase in the bremsstrahlung yield from MWNT is noticed until an intensity of $5 \times 10^{16} \mathrm{~W} \mathrm{~cm}^{-2}$ after which it exhibits a saturation behavior. To illustrate this point, the ratio of the bremsstrahlung yield (blue) from both the samples is plotted in the same graph with a different $y$-axis (given of right side). At higher intensities, the x-ray yield from MWNT sample shows saturation resulting in a steady decline of yield ratio (black open rhombus). This behavior is attributed to the detrimental effect $^{19}$ of the rising edge of the laser pulse on the surface topology. Examining the ablated section under optical microscope reconfirms the fact that the entire coating surface is peeled off from the substrate layer. The enhanced x-ray yield, a direct measure of the "hot" electron population, clearly validates the enhanced laser absorption by the plasma ${ }^{5,21}$ produced on MWNT coated targets. To measure the energy of the hot electron population, bremsstrahlung spectra were recorded at an input laser intensity of $2 \times 10^{16} \mathrm{~W} \mathrm{~cm}^{-2}$. Spectra in the energy range of $50-500 \mathrm{keV}$ were recorded over 4000 independent laser shots keeping photon count rate $<0.1$ per pulse to ensure rejection of "pile up" events. The measurement revealed two distinct hot electron temperature components $8 \pm 1$ and $36 \pm 2 \mathrm{keV}$ for the MWNT samples, whereas only one hot electron temperature of $9 \pm 2 \mathrm{keV}$ was found for polished $\mathrm{Cu}$ (for data on the polished copper targets please refer to our earlier work). ${ }^{5,22}$ The relative yields (integrated from 50 to $500 \mathrm{keV}$ ) of the 6 and $35 \mathrm{keV}$ components are computed and we find that the latter is hundred times larger than the former for carbon nanotube (MWNT) coated substrate. This is easy to understand because the $6 \mathrm{keV}$ component hardly contributes to emission above $50 \mathrm{keV}$.

Let us try to understand the origin of these temperatures. The intense, ultrashort laser pulses instantly ionizes the solid target and is further absorbed by plasma absorption processes-collisional (inverse bremsstrahlung) as well collisionless processes such as resonance absorption ${ }^{23}$ (RA) etc.
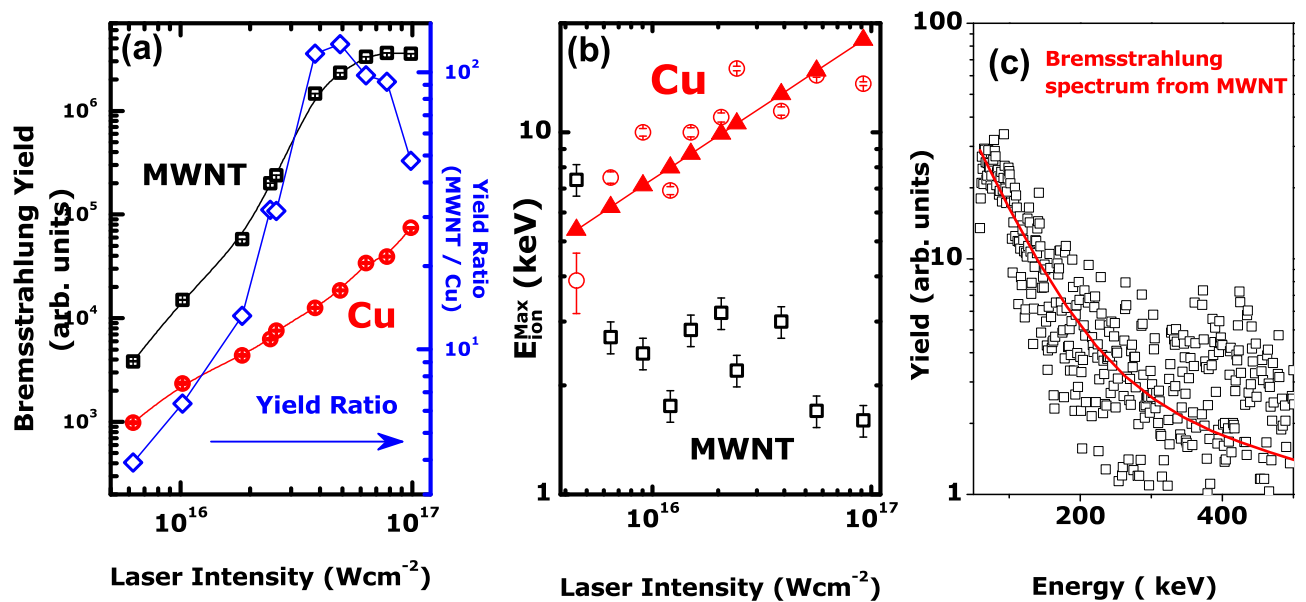

FIG. 2. (Color online) (a) Bremsstrahlung yield recorded from MWNT (black) and $\mathrm{Cu}$ (red) samples with laser intensity. The ratio of the bremsstrahlung yield (blue) from both the samples is plotted in the same graph with a different $y$-axis (right). MWNT sample produced two orders of magnitude larger x-ray flux. (b) shows the maximum ion energies recorded from MWNT (black) and $\mathrm{Cu}$ (red) under identical experimental conditions. The solid line in this figure shows that $\mathrm{Cu}$ ion energy follows $\left(I \lambda^{2}\right)^{0.4}$ scaling. Note that MWNTs do not follow this scaling. (c) Bremsstrahlung spectrum from MWNT. The solid line shows a hot electron component at $36 \mathrm{keV}$. The low energy component is not shown here. The data for the polished $\mathrm{Cu}$ have been reported earlier by us. (See the references, Rajeev et al., 2002 and 2003.) 
At high intensities and high densities, the collisionless process dominates the plasma absorption and depletes most of the input laser energy. RA results from the damping of collective plasma wave excited by input $p$-polarized laser pulse. The absorbed energy is converted into the kinetic energy of hot electrons, called thus because their temperature (typically 10-20 keV even at the modest laser intensities used in this report) is much larger than the energy of the bulk of electrons in the plasma (typically, $100 \mathrm{eV}$ at $10^{15} \mathrm{~W} \mathrm{~cm}^{-2}$ ). These hot electrons can then release energy in the form of bremsstrahlung or characteristic x-rays. The excitation of plasma wave is strongly dependent on the input laser intensity and the hot electron temperature generated by RA can be expressed by the well known scaling law ${ }^{24} T_{\text {hot }}=14\left(T_{c} I \lambda^{2}\right)^{0.33}$ where $T_{\text {hot }}$ and $T_{c}$ are the hot electron temperature and bulk plasma electron temperature expressed in $\mathrm{keV}, I$ is the input laser intensity expressed in units of $10^{16} \mathrm{~W} \mathrm{~cm}^{-2}$, and $\lambda$ the laser wavelength expressed in micrometers. The hot electrons also set up a potential difference that accelerates ions from the freely expanding plasma. ${ }^{25-28}$

The laser pulse shining on a nanotube coated surface experiences a local electric field (and hence light intensity) amplification, simply understood to be due to the well known "lightning rod" effect. ${ }^{5}$ Recently, this enhancement has been modeled in great detail and simple scaling laws have emerged. The local enhancement near the tip of the nanotubes can be simply expressed ${ }^{13}$ as $E_{\text {local }}=1.2(2.15+L / r)^{0.9}$, where $r$ and $L$ are the radius and length of the nanotubes, respectively. In the present case $(r=50 \mathrm{~nm}$ and $L$ $=1100 \mathrm{~nm}$ ), the enhancement factor comes out to be 21 for a single nanotube. However, for an array of nanotubes, the enhancement factor is modified by screening, ${ }^{29}$ the screening factor $F$ given by $[1-0.7 \exp (-0.66 s / L)]$, where $s$ is the average distance between any two neighboring nanotubes and $L$, the length of an individual nanotube. For our case $(s=160 \mathrm{~nm})$, we therefore get a screened local electric field enhancement factor $\left(F \times E_{\text {local }}\right)$ of 7.7.

Let us compare this with the field enhancement indicated by the measured hot electron temperatures. For the MWNT sample this is $36 \mathrm{keV}$ (as compared to $9 \mathrm{keV}$ for polished copper) ${ }^{5,22}$ From the hot electron temperature scaling law mentioned above, using a measured $T_{c}$ of $0.14 \mathrm{keV}$ this would indicate a local intensity enhancement of 58 times for the MWNT, which translates to a local field enhancement of $\approx 7$. Thus we see that the factor estimated purely from electrostatic field enhancement theory matches very well with that obtained from measured bremsstrahlung data. This provides a strong justification for the use of simple electrostatic local field scaling laws (which are valid under the condition that the width of the nanotube is much smaller than the wavelength of the incident light) and also provides confidence that these laws can be used for optimizing the designs of efficient x-ray emitters.

We now examine whether an increase of hot electron energies can directly lead to enhanced energetic ion emission. The maximum ion energies recorded from both the samples under identical experimental conditions are shown in Fig. 2(b). Evidently, the maximum energy of the ions emitted from $\mathrm{Cu}$ sample steadily increases reasonably fol-
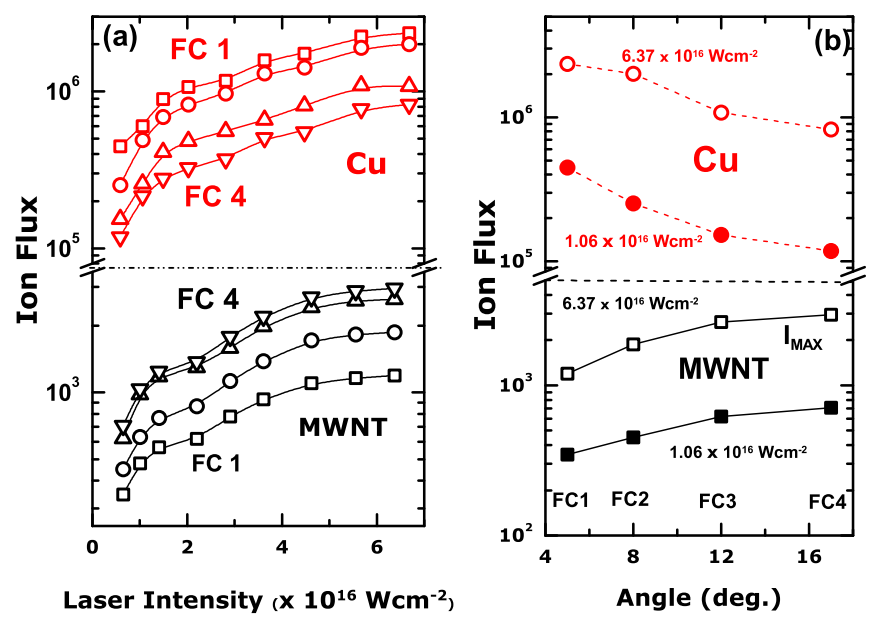

FIG. 3. (Color online) The ion flux recorded by the AnFCs for MWNT (black) and $\mathrm{Cu}$ (red) are shown in (a). Three orders of magnitude reduction in on debris from MWNT sample are evident. Angle resolved ion flux distribution is plotted in (b). With increasing laser intensity, the ion flux for $\mathrm{Cu}$ (red) peaks around the target normal direction whereas, in contradiction, in case of MWNT, the flux become more divergent in nature.

lowing a scaling law behavior of $\approx\left(I \lambda^{2}\right)^{0.4}$ as reported by previous measurements. ${ }^{19,30}$ In comparison, the MWNT sample exhibits a completely contradictory behavior. The maximum ion energies from MWNT sample steadily go down with increasing laser intensity. Notably, except at the lowest laser intensity used in the experiment, the maximum ion energies recorded from the MWNT samples are always lower than the ion energies recorded from $\mathrm{Cu}$ samples.

The ion accelerating electric field in intense, short pulse laser produced plasma can be written ${ }^{31}$ as $E_{\text {accl }}$ $=k_{B} T_{e} / e\left[\max \left(L_{n}, \lambda_{D}\right)\right]$ where, $k_{B}$ is the Boltzmann constant, $T_{e}$ is the hot electron temperature, $\lambda_{D}$ is the Debye length, and $L_{n}$ is the local plasma scale length. Though this model is based on the isothermal free expansion of the laser produced plasmas, it is known to explain the basic features of experimental observations satisfactorily. As we argued above, the electric field of the laser is locally enhanced near the tip of the nanotubes by the lightning rod effect and this results in early plasma formation, thereby creating a modulation in the sheath layer formed by the escaping hot electrons. A modulation in the sheath layer leads to nonplanar plasma expansion $^{21}$ and consequently decreases ion accelerating potential leading to a reduction in emitted ion energies. ${ }^{31,33}$

It is well known that the ion acceleration occurs in a direction normal to the local sheath surface, ${ }^{32}$ so a modulated sheath layer is expected ${ }^{33}$ to give rise to a divergent ion emission which will be manifested in terms of the angular distributions of the emitted ion flux. To estimate the angular divergence of the emitted ion flux from both the surfaces, AnFCs are employed (Fig. 1) at angles of $5^{\circ}, 8^{\circ}, 12^{\circ}$, and $17^{\circ}$ with respect to target normal direction. In Fig. 3(a) we present the ion flux recorded by AnFCs from both surfaces. It is evident that the ion flux recorded by $\mathrm{Cu}$ (red) is three orders of magnitude higher than MWNT (black) over the entire experimental laser intensity range of this experiment. These observations immediately point out that MWNT coated hard x-ray sources would offer the additional advan- 
tage of low debris-an aspect not demonstrated with any other laser excited sources.

Figure 3 also reveals an interesting aspect about the ion angular distributions from both surfaces. For $\mathrm{Cu}$, the innermost AnFC (FC $\left.1,5^{\circ}\right)$ receives the maximum ion flux and the flux density steadily decreases as one goes further away from target normal direction, giving a bell shaped angular distribution similar to previous reports. ${ }^{33}$ A strikingly opposite behavior is observed for MWNT. In this case, the outermost $\mathrm{AnFC}\left(\mathrm{FC} 4,17^{\circ}\right)$ receives the maximum ion flux and as one goes closer to the target normal direction a monotonic reduction in ion flux is observed. To elucidate this contradictory behavior, the ion fluxes recorded from both samples at two different laser intensities $1 \times 10^{16}$ and $6 \times 10^{16} \mathrm{~W} \mathrm{~cm}^{-2}$ are presented in Fig. 3(b). It is to be noted that apart from the resemblance to the bell shaped distribution, the ion flux distribution from $\mathrm{Cu}$ (red color) reveals that the angular distribution of emitted ions peaks along the target normal direction with increasing laser intensity. A contradictory behavior is observed for MWNT (in black color) where the outer FCs receive more ion flux compared to inner ones with increase in laser intensity. This indicates the extremely divergent nature of ion emission from MWNT coated surfaces. At present the exact physics issues that result in the ion reduction that is incongruent with hotter electrons are not very clear. The divergent nature of ion angular distribution does however provide a pointer to the presence of a modulation in plasma sheath layer surface caused by the local enhancement of electric field near the tips of the nanotubes.

In conclusion, we have demonstrated that carbon nanotube coated surfaces excited by moderate intensity laser pulses $\left(10^{15}-10^{16} \mathrm{~W} \mathrm{~cm}^{-2}\right)$ offer two orders of magnitude enhancement in hard $\mathrm{x}$-ray emission in the $50-500 \mathrm{keV}$ region, simultaneously with three orders of magnitude suppression in ion debris. We have demonstrated x-ray enhancement at intensities larger than the lower limit stated above, but it is very likely that future efforts in target optimization can substantially lower the required laser intensities. In addition, kilohertz repetition rate femtosecond lasers that produce intensities in the range of $10^{16}-10^{17} \mathrm{~W} \mathrm{~cm}^{-2}$ are already available commercially paving the way for practical, tabletop hard $\mathrm{x}$-ray sources operating at kilohertz repetition rates. The x-ray dosage from laser produced plasmas with a plain solid target from similar laser powers ${ }^{34}$ was earlier found to be adequate for imaging applications. With a hundredfold enhancement from the MWNT coated target, such imaging applications could only be easier. The enhanced x-ray emission is simply explained by enhancement of local electric fields at the nanotubes. Our study offers clear pointers to the optimization of the physics for generating hotter plasmas and enhancing photon emissions from dense plasmas excited by femtosecond, high intensity lasers.
M.K. thanks DST, Government of India for a Swarnajayanti Fellowship and GRK acknowledges a DAE-SRC-ORI grant.

${ }^{1}$ D. Giulietti and L. A. Gizzi, Riv. Nuovo Cimento 21, 1 (1998).

${ }^{2} \mathrm{P}$. Gibbon, Short Pulse Laser Interactions with Matter (Imperial College Press, London, 2005).

${ }^{3}$ J. D. Kmetec, C. L. Gordon, J. J. Macklin, B. E. Lemoff, G. S. Brown, and S. E. Harris, Phys. Rev. Lett. 68, 1527 (1992).

${ }^{4}$ H. A. Sumeruk, S. Kneip, D. R. Symes, I. V. Churina, A. V. Belolipetski, T. D. Donnelly, and T. Ditmire, Phys. Rev. Lett. 98, 045001 (2007).

${ }^{5}$ P. P. Rajeev, P. Taneja, A. S. Sandhu, P. Ayyub, and G. R. Kumar, Phys. Rev. Lett. 90, 115002 (2003).

${ }^{6}$ M. Anand, S. Kahaly, G. R. Kumar, M. Krishnamurthy, A. S. Sandhu, and P. Gibbon, Appl. Phys. Lett. 88, 181111 (2006).

${ }^{7}$ T. Ditmire, T. Donnelly, R. W. Falcone, and M. D. Perry, Phys. Rev. Lett. 75, 3122 (1995).

${ }^{8}$ Y. L. Shao, T. Ditmire, J. W. G. Tisch, E. Springate, J. P. Marongos, and M. H. R. Hutchinson, Phys. Rev. Lett. 77, 3343 (1996).

${ }^{9}$ V. Kumarappan, M. Krishnamurthy, D. Mathur, and L. C. Tribedi, Phys. Rev. A 63, 023203 (2001).

${ }^{10}$ J. Yu, Z. Jiang, J. C. Kieffer, and A. Krol, Phys. Plasmas 6, 1318 (1999).

${ }^{11}$ S. Iijima, Nature (London) 354, 56 (1991).

${ }^{12}$ P. G. Collins and A. A. Zettl, Appl. Phys. Lett. 69, 1969 (1996).

${ }^{13}$ C. J. Edgcombe and U. Valdre, J. Microsc. 203, 188 (2001).

${ }^{14}$ J. W. Gadzuk and E. W. Plummer, Rev. Mod. Phys. 45, 487 (1973).

${ }^{15}$ A. L. Musatov, K. R. Izrael'yants, A. B. Ormont, A. V. Krestintin, N. A. Kiselev, V. V. Artemov, O. M. Zhigalina, and Y. V. Grigoriev, Appl. Phys. Lett. 87, 181919 (2005).

${ }^{16}$ T. Nishikawa, S. Suzuki, Y. Watanabe, O. Zhou, and H. Nakano, Appl. Phys. B: Lasers Opt. 78, 885 (2004).

${ }^{17}$ O. Zhou and J. P. Lu, U. S. Patent No. 6,553,096 (22 April 2003).

${ }^{18}$ R. Andrews, D. Jacques, A. M. Rao, F. Derbyshire, D. Qian, X. Fan, E. C. Dickey, and J. Chen, Chem. Phys. Lett. 303, 467 (1999).

${ }^{19}$ S. Bagchi, P. P. Kiran, M. K. Bhuyan, S. Bose, P. Ayyub, M. Krishnamurthy, and G. R. Kumar, Appl. Phys. B: Lasers Opt. 88, 167 (2007).

${ }^{20}$ G. F. Knoll, Radiation Detection and Measurement (Wiley, New York, 1989).

${ }^{21}$ S. Bagchi, P. P. Kiran, M. K. Bhuyan, S. Bose, P. Ayyub, M. Krishnamurthy, and G. R. Kumar, Appl. Phys. Lett. 90, 141502 (2007).

${ }^{22}$ P. P. Rajeev, S. Banerjee, A. S. Sandhu, R. C. Issac, L. C. Tribedi, and G. Ravindra Kumar, Phys. Rev. A 65, 052903 (2002).

${ }^{23}$ W. L. Kruer, The Physics of Laser Plasma Interactions (Addison-Wesley, New York, 1988).

${ }^{24}$ D. W. Forslund, J. M. Kindel, and K. Lee, Phys. Rev. Lett. 39, 284 (1977).

${ }^{25}$ P. Mora, Phys. Rev. Lett. 90, 185002 (2003).

${ }^{26}$ R. Decoste and B. H. Ripin, Phys. Rev. Lett. 40, 34 (1978).

${ }^{27}$ L. M. Wickens, J. E. Allen, and P. T. Remsby, Phys. Rev. Lett. 41, 243 (1978).

${ }^{28}$ P. Mora and R. Pellat, Phys. Fluids 22, 2300 (1979).

${ }^{29}$ M. Lim, D. Kim, S. Y. Kim, and J.-E. Bouree, J. Phys. A: Math. Theor. 40, 853 (2007).

${ }^{30}$ E. L. Clark, K. Kruschelnik, M. Zepf, F. N. Beg, M. Tatarakis, M. Machacek, M. I. K. Santala, I. Watts, P. A. Norreys, and A. E. Dangor, Phys. Rev. Lett. 85, 1654 (2000).

${ }^{31}$ S. C. Wilks, A. B. Langdon, T. E. Cowan, M. Roth, M. Singh, S. Hatchett, M. H. Key, D. Pennington, A. Mackinnon, and R. A. Snavely, Phys. Plasmas 8, 542 (2001).

${ }^{32}$ A. Zhidkov, A. Sasaki, and T. Tajima, Phys. Rev. E 61, R2224 (2000).

${ }^{33}$ M. Roth, A. Blazevic, M. Geissel, T. Schlegel, T. E. Cowan, M. Allen, J. C. Gauthier, P. Audebert, J. Fuchs, J. Meyer-ter-Vehn, M. Hegelich, S. Karsch, and A. Pukhov, Phys. Rev. ST Accel. Beams 5, 061301 (2002).

${ }^{34}$ S. Svanberg, Meas. Sci. Technol. 12, 1777 (2001). 